\title{
Measurements of Heavy Flavor Single Leptons by PHENIX
}

\author{
Donald Hornback (for the PHENIX Collaboration) * \\ University of Tennessee - Department of Physics and Astronomy \\ Knoxville, TN 37996, USA
}

\begin{abstract}
PHENIX has measured single leptons in both $\mathrm{p}+\mathrm{p}$ and $\mathrm{Au}+\mathrm{Au}$ collisions at $\sqrt{s_{N N}}=$ $200 \mathrm{GeV}$. Measurements in $\mathrm{p}+\mathrm{p}$ collisions permit a determination of the total charm production cross section which can be compared to pQCD predictions. Heavy flavor production in $\mathrm{Au}+\mathrm{Au}$ serves as a tool for studying the dense partonic matter produced in $\mathrm{Au}+\mathrm{Au}$ collisions that can be interpreted through the simultaneous measurement of the azimuthal anisotropy parameter $v_{2}\left(p_{T}\right)$ and the nuclear modification factor $\mathrm{R}_{A A}\left(p_{T}\right)$. In the context of existing predictions, the observed flow and energy loss of heavy quarks, in addition to that already seen for light mesons, suggests that the matter formed in $\mathrm{Au}+\mathrm{Au}$ collisions at RHIC is a near-perfect fluid.
\end{abstract}

\section{$1 \quad$ Studying heavy flavor production with single leptons}

The measurement of single leptons at high transverse momentum $\left(p_{T} \gtrsim 1.0 \mathrm{GeV} / \mathrm{c}\right)$, specifically electrons and muons which arise from the semi-leptonic decay of heavy flavor hadrons, play an important role in the study of heavy-quark production. Bottom and charm quarks are expected to have sufficient mass to permit next-to-leading order perturbative QCD (NLO pQCD) calculations to describe their production cross sections in $\mathrm{p}+\mathrm{p}$ collisions at high $p_{T}$. Bottom production at the Tevatron [3] is shown to be well described by such calculations, charm production cross sections are determined to be $\approx 50 \%$ higher than theory, though compatible within the experimental and theoretical uncertainties [4]. The PHENIX single electron results presented here provide improved comparisons to theoretical calculations through both extension to higher $p_{T}$ and reduced experimental uncertainties. The single electron $\mathrm{p}+\mathrm{p}$ measurement also serves as a baseline for the most recent single electron measurements in $\mathrm{Au}+\mathrm{Au}$ collisions. Azimuthal anisotropies observed in the collective motion of charm quarks in the produced medium have interesting implications for the properties of the medium and is further discussed in section 4. PHENIX also possesses the capability to measure single muons at forward rapidity. Although such measurements are currently underway within PHENIX, the single electron measurements are well established with clear physics messages. Discussion in these proceedings are therefore restricted to single electron results.

\section{PHENIX Experiment and analysis}

PHENIX measures single electrons with two separate central arms, each with $90^{\circ}$ azimuthal acceptance and pseudorapidity coverage of $|\eta|<0.35$. Electrons are measured in the central arms using combined information from an electromagnetic calorimeter and a ring imaging Cerenkov detector. Muons are measured using two separate muon spectrometers with azimuthal coverage and covering the pseudorapidity range $1.2<|\eta|<2.4$. Muons are

*For the full PHENIX collaboration author list, see Ref. [2] 
identified in the forward and backward directions through the use Iarocci tubes interleaved between steel absorber plates. Precise muon momentum determination is accomplished using cathode strip chambers inside a magnetic field. For more details on the PHENIX experiment, please see [5].

The identification of single electrons from heavy flavor is performed through the statistical subtraction of background sources, with the remaining lepton signal being attributed to open heavy flavor decay. This technique was first applied at the $I S R$ at CERN, which measured single electrons from heavy flavor production in $\mathrm{p}+\mathrm{p}$ collisions at $\sqrt{s}=30-63 \mathrm{GeV}$ [6]. PHENIX applies two independent background estimates, referred to as the converter and cocktail methods, which serve as experimental cross checks, allowing for a reduction in the systematic uncertainties associated with the extracted single electron production cross section [7].

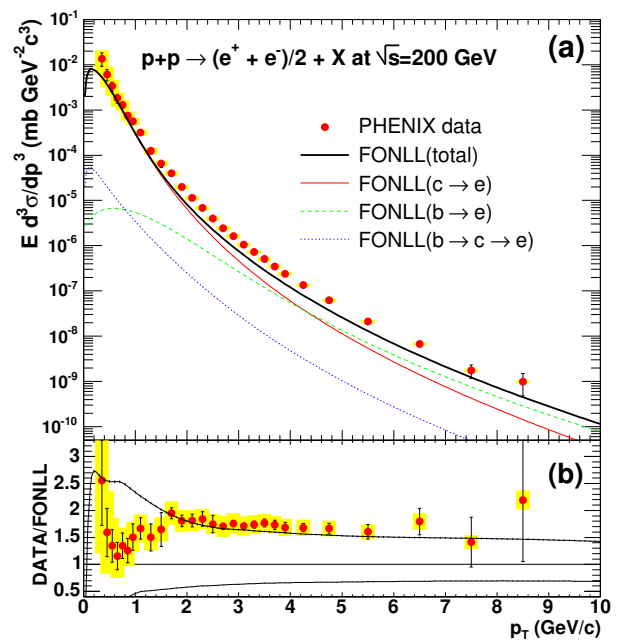

Figure 1: (a) Transverse momentum distribution of single electron production cross section compared to FONLL [8] calculation. (b) Ratio of single electron cross section to FONLL.

\section{Open heavy-flavor production in $\mathrm{p}+\mathrm{p}$}

At all transverse momentum the dominant source of background is from $\pi^{0}$ decay, directly from Dalitz decay or indirectly from photon conversion. PHENIX constrains the $\pi^{0}$ spectrum by direct measurement. For the convertor method a thin brass sheet $\left(1.67 \% X_{0}\right)$ of known thickness is introduced around the beam pipe for a fraction of the total run time. This material serves to increase electrons from photon conversion by a fixed amount. Through a comparison of run periods with and without the additional conversion material, the yield of photonic electrons is accurately measured, allowing for the extraction of the heavy-flavor electron signal down to a $p_{T}=0.3 \mathrm{GeV} / c$. The "cocktail" method calculates various electron sources collectively using a Monte Carlo event generator of hadron decays. The converter method also serves to calibrate the backgrounds as estimated by the cocktail method. The 
convertor method is statistically limited to low $p_{T}$ due to the short run time devoted to its use. The cocktail method allows for extraction of the heavy flavor signal up to the current statistical limitations of the data, $p_{T}=9.0 \mathrm{GeV} / c$. These analysis techniques are applied to both the $\mathrm{p}+\mathrm{p}$ and $\mathrm{Au}+\mathrm{Au}$ measurements presented.

The production cross section of single electrons from heavy-flavor decay as measured by PHENIX is shown in Fig. 1. Figure. 1(a) shows the invariant differential cross section of single electrons compared to the central values of a Fixed Order Next to Leading Log (FONLL) [8] pQCD calculation. Relative contributions from charm and bottom are also shown, with bottom overtaking charm for $p_{T}>4.0 \mathrm{GeV} / c$. The ratio of data to FONLL is shown in Fig. 1b. The theoretical upper and lower limit band is shown. Some small $p_{T}$ dependence is observed in this ratio for $p_{T}<2.0 \mathrm{GeV} / c$. Approximating this ratio as nearly constant by fitting with a line over $0.3<p_{T}<9.0 \mathrm{GeV} / c$ yields a ratio of $1.72 \pm 0.02^{\text {stat }}$ $\pm 0.19^{\text {sys }}$. This is similar to ratios observed in charm production at the Tevatron [4]. As seen in the lower plot, the data lies along the upper theoretical uncertainty band. The total charm cross section is determined to be $\sigma_{c \bar{c}}=567 \pm 57^{\text {stat }} \pm 224^{\text {sys }} \mu b$.

\section{Open heavy flavor production in $\mathrm{Au}+\mathrm{Au}$ collisions}

Single electrons from heavy flavor semi-leptonic decay in $\mathrm{Au}+\mathrm{Au}$ collisions have also been measured by PHENIX [2]. The suppression of light hadron production at high $p_{T}$ has previously been observed in $\mathrm{Au}+\mathrm{Au}$ collisions at $\mathrm{RHIC}$ and is taken to be caused by partonic energy loss in the produced medium. Energy loss by heavy flavor quarks is expected to be less than that for light quarks due to reduced gluon radiation in the forward direction, commonly referred to as the dead-cone effect [9]. The extent of energy loss in the medium can be quantified using the nuclear modification factor, $R_{A A}$, which is the ratio of the yield of single electrons in $\mathrm{Au}+\mathrm{Au}$ collisions for a given centrality class divided by a $\mathrm{p}+\mathrm{p}$ reference scaled by the number of binary collisions for $\mathrm{Au}+\mathrm{Au}$. By construction, $R_{A A}=1$ for processes that scale by the number of underlying nucleon-nucleon collisions.

Figure 2 shows three different plots of $R_{A A}$ as a function of the number of participants, $N_{\text {part }}$. The solid circles represent the integrated heavy-flavor single electrons for $p_{T}>0.3$ $\mathrm{GeV} / c$, with each data point corresponding to a particular centrality class. $R_{A A}$ does not deviate from unity for all centralities confirming the expectation that the total heavy-flavor yield follows binary scaling. The solid squares show $R_{A A}$ for $\pi^{0}$ with $p_{T}>4.0 \mathrm{GeV} / c$, which exhibit the previously observed phenomena of increasing suppression with increasing centrality [10]. The open circles represent heavy-flavor single electron yield, plotted for $p_{T}$ $>3.0 \mathrm{GeV} / c$ instead of $4.0 \mathrm{GeV} / c$, since electrons from charm decay originate primarily from $\mathrm{D}$ mesons of $p_{T}>4.0 \mathrm{GeV} / c$. Counter to original expectations, heavy-flavor does exhibit clear high $p_{T}$ suppression which increases smoothly with $N_{\text {part }}$, though the extent of suppression is somewhat less than that observed for the light quark $\pi^{0}$ meson. This suppression of heavy-flavor yields at high $p_{T}$ suggests a strong coupling of heavy quarks to the partonic medium produced in $\mathrm{Au}+\mathrm{Au}$ collisions [2].

This measurement has further implications when coupled with the observed elliptic flow of heavy flavor. This flow is azimuthal anisotropy characterized by the parameter $v_{2}$ which is the coefficient of the second harmonic of the Fourier expansion of the azimuthal distribution with respect to the reaction plane [11]. Figure 3 shows the PHENIX measurement of heavyflavor single electron $v_{2}$ values (solid circles) for minimum bias $\mathrm{Au}+\mathrm{Au}$ collisions. The data indicate a non-zero $v_{2}$ value for charm quarks, indicating that they participate in the 


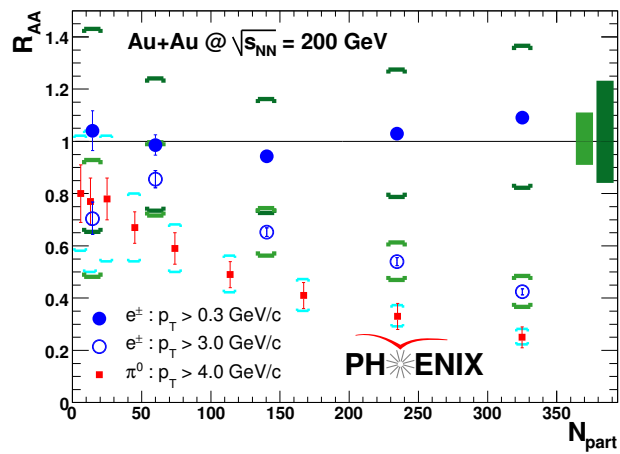

Figure 2: Nuclear modification factor, $R_{A A}$ (defined in text) as a function of the number of participant, $N_{\text {part }}$ for single electrons in $\mathrm{Au}+\mathrm{Au}$ collisions at $\sqrt{s_{N N}}=200 \mathrm{GeV}$ for $\mathrm{e}^{ \pm}$and $\pi^{0}$.

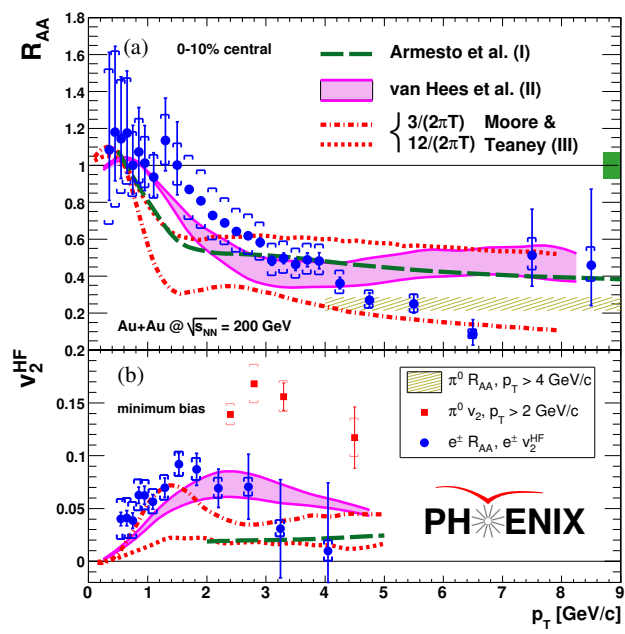

Figure 3: Comparison of measured $R_{A A}$ and $v_{2}$ as a function of transverse momentum in $\mathrm{Au}+\mathrm{Au}$ collisions with hydrodynamical models.

collective flow of the medium produced at RHIC. Most strikingly, hydrodynamical models that describe both the high $p_{T}$ suppression and elliptic flow [citations] suggest that the medium's viscosity resides near the conjectured quantum lower bound, making the medium produced in $\mathrm{Au}+\mathrm{Au}$ collisions at RHIC a near perfect fluid.

\section{Acknowledgments}

This research is sponsored by the Division of Nuclear Physics , U.S. Department of Energy, under contract DE-FG02-96ER40982 with the University of Tennessee. The complete list of PHENIX acknowledgments is provided in Ref. [7].

\section{References}

[1] Slides: http: //indico. cern. ch/contributionDisplay. py? contribId=198\&sessionId=5\&conf Id=9499

[2] A. Adare et al. Phys. Rev. Lett., 98, 2007.

[3] Cacciari M. hep-ph/0407187, 2004.

[4] D. Acosta et al. Phys. Rev. Lett., 91:241804, 2003.

[5] K. Adcox et al. Nucl. Instrum. Meth. A, 499:469-602, 2003.

[6] Perez P. Phys. Lett., B112:260, 1976.

[7] A. Adare et al. Phys. Rev. Lett., 97, 2006.

[8] Cacciari M. Phys. Rev. Lett., 95:122001, 2005.

[9] Dokshitzer Y. and Kharzeev D. Phys. Lett. B, 519:199-206, 2001.

[10] A. Adare et al. Phys. Rev. Lett., 96, 2006.

[11] Wong C.Y. Phys. Lett., 88B:39, 1979. 